\title{
HILBERT 90 FOR BIQUADRATIC EXTENSIONS
}

\author{
ROMAN DWILEWICZ, JÁN MINÁČ, ANDREW SCHULTZ, AND JOHN \\ SWALLOW
}

\author{
Here something stubborn comes, \\ Dislodging the earth crumbs \\ And making crusty rubble. \\ It comes up bending double, \\ And looks like a green staple. \\ It could be seedling maple, \\ Or artichoke, or bean. \\ That remains to be seen. \\ -Richard Wilbur, "Seed Leaves" (1-8)
}

Whether reading or writing mathematics, it is over the simplest theorems that we linger. Something quickens at the hope that such a theorem, a result of already uncommon brevity, might grow into a result of uncommon grandeur. Might it become still more wondrous, maturing into a figure as elegant as it is encompassing?

Hilbert's Theorem 90 is just such a theorem. In its simplest form, in the case of a quadratic extension of fields, Hilbert's Theorem 90 is a seedling, and turning it over in our hands, we naturally speculate about the mature form we might one day behold.

Most commonly, friends tell us to expect from the seed what is affectionately known as "Hilbert 90": a result in the theory of cyclic extensions. Those who have surveyed Galois cohomology, however, know that A. Speiser sensed something different, a cohomological result in a non-cohomological era. Recognizing the power of this result, E. Noether put it to use, and the result became known as Noether's Theorem. More recently, others have imagined the mature form being

The first author was partially supported by a University of Missouri Research Board grant, and by Polish Committee for Scientific Research grant KBN2P03A04415. The second author was supported in part by Natural Sciences and Engineering Research Council of Canada grant R0370A01, and by a Distinguished Professorship for 2004-2005 at the University of Western Ontario. 
different still: the spectacular announcement by Voevodsky of the validity of the Bloch-Kato Conjecture contains, in fact, a generalization of Hilbert's Theorem 90 to Milnor $K$-theory.*

We propose to linger long enough to investigate the very first shoots of the generalization from quadratic to biquadratic field extensions. The quadratic case is well-known and already significant. The biquadratic case, particularly the difference between it and the quadratic case, appears new. Studying the leaves quite closely, we will encounter the possibility that the leaves unfurl in a different fashion, something between the traditional result on cyclic extensions and Speiser's and Noether's result in cohomology. Exploring this new possibility, we apprehend connections among multiplicative groups of fields, values of binary quadratic forms, and even a bit of module theory over a group ring.

\section{Artichoke, or Bean?: The Quadratic Seed}

The traditional form of Hilbert 90 states that the kernel of the norm operator for a cyclic extension is as small as possible, a statement we make precise below in the context of a quadratic extension.

Let $F$ be a field of characteristic not 2. Then any quadratic extension $L$ of $F$ has the form $L=F(\sqrt{a})$, for a suitable element $a \in F \backslash F^{2}$, where $F^{2}$ denotes the set of squares in $F$. Each element $l \in F(\sqrt{a})$ may be written uniquely as $l=f_{1}+f_{2} \sqrt{a}$ with $f_{1}, f_{2} \in F$. The Galois group $G:=\operatorname{Gal}(L / F)$ consists of two elements: the identity automorphism, denoted as 1 , and $\sigma$, which maps $l$ to $\sigma(l)=f_{1}-f_{2} \sqrt{a}$. The norm function $N_{L / F}: L \rightarrow F$ is defined by the formula $N_{L / F}(l)=l \cdot \sigma(l)=$ $f_{1}^{2}-a f_{2}^{2}$, and it restricts to a morphism on the multiplicative group $L^{\times}:=L \backslash\{0\}$. Since there is no chance for confusion, we write $N_{L / F}$ for this restricted function.

It is natural to determine the kernel of this morphism. Since $N(l)=$ $l \cdot \sigma(l)=N(\sigma(l))$ for each $l \in L^{\times}$, we see that elements of the form

${ }^{*}$ The interested reader should consult $[\mathrm{LeSc}$ and $\mathrm{H}$ ] for some initial background on Hilbert's Theorem 90 and We page 30] for its cohomological generalization. To observe the use of Hilbert 90-type theorems in the partially published work of Rost and Voevodsky on the Bloch-Kato Conjecture, see V1, V2]. For further original sources on Hilbert 90 and its cohomological generalization, see Kummer's early discovery of a special case [K], followed by Speiser's result [S] and Noether's application $[\mathrm{N}]$. 
$\frac{l}{\sigma(l)}$ certainly lie in ker $N_{L / F}$. Hilbert's Theorem 90 says that ker $N_{L / F}$ contains no other elements.

Theorem 1 (Hilbert 90 for $F(\sqrt{a}) / F)$.

$$
\operatorname{ker}\left(N_{F(\sqrt{a}) / F}: F(\sqrt{a})^{\times} \rightarrow F^{\times}\right)=\left\{\frac{l}{\sigma(l)} \mid l \in F(\sqrt{a})^{\times}\right\} .
$$

Not surprisingly, this simple statement is already important. For example, the classical parameterization of Pythagorean triples is a beautiful consequence of this statement. The idea of the proof can be traced back to O. Taussky [T, pages 808-809] (who was in fact a co-editor of Hilbert's collected works), and Elkies independently discovered the proof in a short, attractive note $[\mathrm{E}]$. See also T. Ono's book [O, pages $4-5]$.

Proof of Theorem 11. Let $t \in \operatorname{ker} N_{L / F}$. If $t=-1$, then $-1=t=\frac{\sqrt{a}}{\sigma(\sqrt{a})}$. Assume therefore that $t \neq-1$. Set $l=1+t$. Then

$$
\sigma(l)=1+\sigma(t)=\sigma(t) t+\sigma(t)=\sigma(t)(t+1)=\sigma(t) l .
$$

Hence $\frac{\sigma(l)}{l}=\sigma(t)$. Applying $\sigma$ to both sides and remembering that $\sigma^{2}=1$ we obtain $\frac{l}{\sigma(l)}=t$, as required. Therefore

$$
\operatorname{ker} N_{L / F} \subseteq\left\{\frac{l}{\sigma(l)} \mid l \in L^{\times}\right\}
$$

We have already observed the reverse inclusion.

The generalization of this result to cyclic extensions, that is, field extensions whose Galois group is generated by a single element, is wellknown, with many finding it quite commonly in the fields they amble through. But might this seed originate something different, something similar but not the same? Something, as our naturalist friends might say, homologous?

Let us review our example above and observe that the seed might be that of an elegant result in Galois cohomology. Consider a map $f: G \rightarrow L^{\times}$satisfying

$$
f\left(\gamma_{1} \gamma_{2}\right)=\left(\gamma_{1} f\left(\gamma_{2}\right)\right) f\left(\gamma_{1}\right), \quad \gamma_{1}, \gamma_{2} \in G .
$$

Because of its similarity to a homomorphism, such a map is called a crossed homomorphism. From the equality $f(1)=(1(f(1))) f(1)=$ $f(1)^{2}$ we deduce that $f(1)=1$. As $G$ is especially simple in our context, 
it follows that a crossed homomorphism will be determined by $f(\sigma)$. From

$$
1=f\left(\sigma^{2}\right)=(\sigma f(\sigma)) f(\sigma)
$$

we see that $f(\sigma) \in \operatorname{ker} N_{L / F}$. Conversely, choosing any element $a$ from ker $N_{L / F}$, we may define a crossed homomorphism $f: G \rightarrow L^{\times}$by $f(1)=1, f(\sigma)=a$. Using Theorem 1, we have $f(\sigma)=l / \sigma(l)$ for some $l \in L^{\times}$. In particular we see that for any crossed homomorphism $f$ there exists $l \in L^{\times}$so that $f(g)=\frac{l}{g(l)}$ for all $g \in G$.

For an arbitrary Galois group $\mathcal{G}=\operatorname{Gal}(\mathcal{L} / \mathcal{F})$, a map $f$ such that $f(g)=\frac{\lambda}{g(\lambda)}$ for some fixed $\lambda \in \mathcal{L}^{\times}$is called a coboundary. Such a map is easily seen to be a crossed homomorphism. The first cohomology group $H^{1}\left(\mathcal{G}, \mathcal{L}^{\times}\right)$of $\mathcal{G}$ with coefficients in $\mathcal{L}^{\times}$is defined to be the group of crossed homomorphisms modulo the subgroup of coboundaries:

$$
H^{1}\left(\mathcal{G}, \mathcal{L}^{\times}\right)=\frac{\{\text { crossed homomorphisms }\}}{\{\text { coboundaries }\}} .
$$

Our result above for the quadratic extension $L / F$ is then equivalent to $H^{1}\left(G, L^{\times}\right)=\{1\}$. Speiser sensed in the seed this elegant outcome, and he proved that $H^{1}\left(\mathcal{G}, \mathcal{L}^{\times}\right)=\{1\}$ for all Galois extensions $\mathcal{L} / \mathcal{F}$ with group $\mathcal{G}$.

\section{The Stalk Unbends: Biquadratic Extensions}

Could not we imagine the seed maturing differently still, as $G$ ever so slightly enlarges? Might we imagine that shooting from the seed is something homologous to both the traditional result on the kernel of the norm operator acting on a cyclic extension as well as Speiser's cohomological theorem?

We consider here the case of a Klein 4-group. Let $E / F$ be a Galois extension with $G:=\operatorname{Gal}(E / F)=\mathbb{Z} / 2 \mathbb{Z} \times \mathbb{Z} / 2 \mathbb{Z}$. Since we assume that $F$ has characteristic not 2 , there exist $a_{1}, a_{2} \in F^{\times}$such that $E=F\left(\sqrt{a_{1}}, \sqrt{a_{2}}\right)$, and $G$ is a group generated by two automorphisms $\sigma_{1}, \sigma_{2} \in G$ such that

$$
\frac{\sigma_{i}\left(\sqrt{a_{j}}\right)}{\sqrt{a_{j}}}=(-1)^{\delta_{i j}}
$$


where $\delta_{i j}$ is Kronecker's delta function: $\delta_{i j}=0$ if $i=j$ and 1 if $i \neq j$. The lattice of subfields of $E$ is then

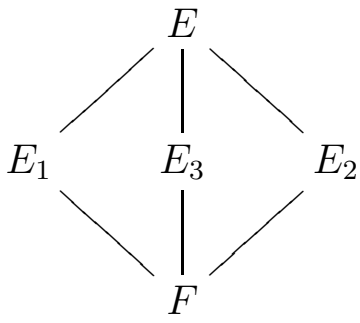

where $E_{1}=F\left(\sqrt{a_{1}}\right), E_{2}=F\left(\sqrt{a_{2}}\right)$, and $E_{3}=F\left(\sqrt{a_{1} a_{2}}\right)$.

Given Hilbert 90 for the two quadratic extensions $E / E_{1}$ and $E / E_{2}$, we shall show $H^{1}\left(G, E^{\times}\right)=\{1\}$ is equivalent to another condition, itself very much like the traditional statement of Hilbert 90. In fact, we shall show that this condition is the "difference" between Speiser's result for biquadratic extensions and Hilbert 90 for quadratic extensions: by adding the new result to Hilbert 90 for quadratic extensions, we obtain Speiser's result in the biquadratic case. It is the new growth from the seed when the cyclic group of order two divides itself into the Klein 4-group.

Some corollaries of this condition have been rediscovered several times, but the connection with Hilbert 90 and the very natural proof below seem new. To establish this equivalence, we rephrase the condition $H^{1}\left(G, E^{\times}\right)=\{1\}$ in the language of elements of the multiplicative group $E^{\times}$.

Let $f: G \rightarrow E^{\times}$be a crossed homomorphism. Since $f(1)=1$ and $f\left(\sigma_{1} \sigma_{2}\right)=\left(\sigma_{1} f\left(\sigma_{2}\right)\right) f\left(\sigma_{1}\right)$ we see that $f$ is determined by its values $\alpha_{i}=f\left(\sigma_{i}\right)$. We also observe

$$
1=f\left(\sigma_{i}^{2}\right)=\left(\sigma_{i}\left(\alpha_{i}\right)\right) \alpha_{i}=N_{E / E_{i}}\left(\alpha_{i}\right) \text { for } i=1,2
$$

and

$$
\left(\sigma_{1}\left(\alpha_{2}\right)\right) \alpha_{1}=f\left(\sigma_{1} \sigma_{2}\right)=f\left(\sigma_{2} \sigma_{1}\right)=\left(\sigma_{2}\left(\alpha_{1}\right)\right) \alpha_{2} .
$$

Conversely, one may readily check that given any elements $\alpha_{1}, \alpha_{2} \in E^{\times}$ such that $N_{E / E_{i}}\left(\alpha_{i}\right)=1, i=1,2$, and $\left(\sigma_{1}\left(\alpha_{2}\right)\right) \alpha_{1}=\left(\sigma_{2}\left(\alpha_{1}\right)\right) \alpha_{2}$, there exists a unique crossed homomorphism $f: G \rightarrow E^{\times}$such that $f\left(\sigma_{i}\right)=$ $\alpha_{i}, i=1,2$.

Since $H^{1}\left(G, E^{\times}\right)=\{1\}$, we also know that for a given crossed homomorphism $f$ there exists $\beta \in E^{\times}$such that $f(g)=\frac{\beta}{g(\beta)}$ for any $g \in G$. 
In particular we have

$$
\alpha_{i}=f\left(\sigma_{i}\right)=\frac{\beta}{\sigma_{i}(\beta)}
$$

for $i=1,2$. Therefore the cohomological identity $H^{1}\left(G, E^{\times}\right)=\{1\}$ may be reformulated as

Theorem 2. Two elements $\alpha_{1}, \alpha_{2} \in E^{\times}$satisfy

(1) $N_{E / E_{1}}\left(\alpha_{1}\right)=N_{E / E_{2}}\left(\alpha_{2}\right)=1$

(2) $\alpha_{1} \cdot \sigma_{1}\left(\alpha_{2}\right)=\alpha_{2} \cdot \sigma_{2}\left(\alpha_{1}\right)$

if and only if there exists $\beta \in E^{\times}$such that $\alpha_{i}=\frac{\beta}{\sigma_{i}(\beta)}$.

This result can be found in [C, page 756].

Theorem 2 shares with Hilbert 90 a concern with the kernel of operators being suitably minimal. However, since it describes the simultaneous vanishing of norms from different quadratic extensions, it is best to think of Theorem 2 as a version of Hilbert 90 with compatibility. We want to see just how far this result reaches above the seed-Hilbert 90 for quadratic extensions - and we show that, assuming Hilbert 90 for quadratic extensions, Theorem 2 is equivalent to a statement that the kernel of a particular operator is minimal.

In order to formulate this result, it is convenient to view $E^{\times}$as a $\mathbb{Z}[G]$-module. Recall the definition of the group ring $\mathbb{Z}[G]$ :

$$
\mathbb{Z}[G]=\left\{c_{0}+c_{1} \sigma_{1}+c_{2} \sigma_{2}+c_{3} \sigma_{1} \sigma_{2} \mid \quad c_{i} \in \mathbb{Z}\right\} .
$$

Then the action of $\mathbb{Z}[G]$ on $E^{\times}$extends, in a natural way, the action of $\sigma_{i}$ on $E^{\times}$:

$$
\left(c_{0}+c_{1} \sigma_{1}+c_{2} \sigma_{2}+c_{3} \sigma_{1} \sigma_{2}\right) \cdot \gamma:=\gamma^{c_{0}} \sigma_{1}(\gamma)^{c_{1}} \sigma_{2}(\gamma)^{c_{2}} \sigma_{1} \sigma_{2}(\gamma)^{c_{3}} .
$$

Viewed this way, multiplication in $E^{\times}$is now achieved by addition in $\mathbb{Z}[G]$, and hence we shall denote $1 \in E^{\times}$as 0 . Observe that $\left(1+\sigma_{1}\right) \gamma$, for instance, means $\gamma \cdot \sigma_{1}(\gamma)=N_{E / E_{1}} \gamma$.

Theorem 3. Given Hilbert 90 for quadratic extensions, Theorem 2 is equivalent to

$$
\operatorname{ker}\left(1-\sigma_{1}\right)\left(1-\sigma_{2}\right)=\operatorname{ker}\left(1-\sigma_{1}\right)+\operatorname{ker}\left(1-\sigma_{2}\right)
$$

REMARK. It may appear that we are viewing this result from one particular direction, specifying the two elements of $G$ as we have. But 
Theorem 2 implies every version of Equation 1 obtained by substituting two generators of $G$ for $\sigma_{1}, \sigma_{2}$. Since the conjunction of any version of Equation 11 with Hilbert 90 for quadratic extensions implies Theorem 2, the particular "difference" we find in Theorem 3 depends only on our vantage point.

Proof. Assume first Hilbert 90 for $E / E_{1}$ and $E / E_{2}$ and Equation 1 . For a fixed $\beta \in E^{\times}$it is clear that $\alpha_{i}=\left(1-\sigma_{i}\right) \beta, i=1,2$, satisfy conditions (1) and (2) of Theorem 2. Hence assume that $\alpha_{1}, \alpha_{2} \in E^{\times}$ are given with $N_{E / E_{i}} \alpha_{i}=1$ and $\left(1-\sigma_{2}\right) \alpha_{1}=\left(1-\sigma_{1}\right) \alpha_{2}$. By Hilbert 90 there exist $\beta_{1}, \beta_{2} \in E^{\times}$such that $\alpha_{i}=\left(1-\sigma_{i}\right) \beta_{i}$. Then consider

$$
0=\left(1-\sigma_{2}\right) \alpha_{1}-\left(1-\sigma_{1}\right) \alpha_{2}=\left(1-\sigma_{2}\right)\left(1-\sigma_{1}\right)\left(\beta_{1}-\beta_{2}\right) .
$$

Hence $\beta_{1}-\beta_{2} \in \operatorname{ker}\left(1-\sigma_{1}\right)\left(1-\sigma_{2}\right)=\operatorname{ker}\left(1-\sigma_{1}\right)+\operatorname{ker}\left(1-\sigma_{2}\right)$. Thus there exist $\epsilon_{i} \in \operatorname{ker}\left(1-\sigma_{i}\right)$ such that $\beta_{1}-\beta_{2}=\varepsilon_{2}-\varepsilon_{1}$, and we set

$$
\beta:=\beta_{1}+\varepsilon_{1}=\beta_{2}+\varepsilon_{2} .
$$

Therefore

$$
\left(1-\sigma_{i}\right) \beta=\left(1-\sigma_{i}\right) \beta_{i}=\alpha_{i} \text { for both } i=1,2 \text {. }
$$

Now assume Theorem 2. If $\gamma=\gamma_{1}+\gamma_{2}$ where $\gamma_{i} \in \operatorname{ker}\left(1-\sigma_{i}\right)$, then

$$
\left(1-\sigma_{1}\right)\left(1-\sigma_{2}\right) \gamma=\left(1-\sigma_{2}\right)\left(1-\sigma_{1}\right) \gamma_{1}+\left(1-\sigma_{1}\right)\left(1-\sigma_{2}\right) \gamma_{2}=0,
$$

providing the inclusion $\operatorname{ker}\left(1-\sigma_{1}\right)+\operatorname{ker}\left(1-\sigma_{2}\right) \subseteq \operatorname{ker}\left(1-\sigma_{1}\right)\left(1-\sigma_{2}\right)$.

To verify the more interesting opposite inclusion, choose $\gamma \in \operatorname{ker}(1-$ $\left.\sigma_{1}\right)\left(1-\sigma_{2}\right)$. Set $\alpha_{1}=\left(1-\sigma_{1}\right) \gamma$ and $\alpha_{2}=0$. Then

$$
N_{E / E_{1}} \alpha_{1}=\left(1+\sigma_{1}\right)\left(\left(1-\sigma_{1}\right) \gamma\right)=0=N_{E / E_{2}} \alpha_{2} .
$$

Also

$$
\left(1-\sigma_{2}\right) \alpha_{1}-\left(1-\sigma_{1}\right) \alpha_{2}=\left(1-\sigma_{2}\right)\left(1-\sigma_{1}\right) \gamma=0 .
$$

Therefore using Theorem 2 we see there exists $\beta \in E^{\times}$such that $\alpha_{1}=$ $\left(1-\sigma_{1}\right) \gamma=\left(1-\sigma_{1}\right) \beta$ and $\alpha_{2}=\left(1-\sigma_{2}\right) \beta$. Hence $\gamma-\beta \in \operatorname{ker}\left(1-\sigma_{1}\right)$ and $\beta \in \operatorname{ker}\left(1-\sigma_{2}\right)$, so that $\gamma=(\gamma-\beta)+\beta \in \operatorname{ker}\left(1-\sigma_{1}\right)+\operatorname{ker}\left(1-\sigma_{2}\right)$.

REMARK. Since $\operatorname{ker}\left(1-\sigma_{1}\right)+\operatorname{ker}\left(1-\sigma_{2}\right) \subseteq \operatorname{ker}\left(1-\sigma_{1}\right)\left(1-\sigma_{2}\right)$ for all $\mathbb{Z}[G]$-modules, Equation 1 says $\operatorname{ker}\left(1-\sigma_{1}\right)\left(1-\sigma_{2}\right)$ is as small as possible. 


\section{3. ". . . And Starts to Ramify": A Form Revealed}

Now we prove Equation 11 directly and observe several corollaries of the result. One corollary, in particular, has been rediscovered several times under a certain attractive disguise as a statement on quadratic binary forms. Although several ingenious and beautiful proofs of this statement have been obtained in the literature, the proof we offer may be the most transparent.

Theorem 4. Suppose that $E / F$ is a biquadratic extension and $G=$ $\operatorname{Gal}(E / F)$ as above. Then the following sets are identical.

(1) $\operatorname{ker}\left(1-\sigma_{1}\right)\left(1-\sigma_{2}\right)$

(2) $\operatorname{ker}\left(1-\sigma_{1}\right)+\operatorname{ker}\left(1-\sigma_{2}\right)$

(3) $\left\langle E_{1}^{\times}, E_{2}^{\times}\right\rangle$

(4) $\left\{e \in E^{\times} \mid N_{E / E_{3}}(e) \in F^{\times}\right\}$

(5) $\left\{e \in E^{\times} \mid N_{E / E_{3}}(e) \in N_{E_{1} / F}\left(E_{1}^{\times}\right) N_{E_{2} / F}\left(E_{2}^{\times}\right)\right\}$.

In the third item $\left\langle E_{1}^{\times}, E_{2}^{\times}\right\rangle$denotes the smallest subgroup containing $E_{1}^{\times}$and $E_{2}^{\times}$. In the last item the product of norm groups denotes the set of all products of elements of the first set with the second.

Taking $N_{E / E_{3}}$ of the sets described in (4) and (5) we obtain

$$
N_{E / E_{3}}\left(E^{\times}\right) \cap F^{\times} \subset N_{E_{1} / F}\left(E_{1}^{\times}\right) N_{E_{2} / F}\left(E_{2}^{\times}\right) .
$$

The reverse inequality follows from the proof of Theorem 4 below, since for each $\gamma_{i} \in E_{i}^{\times}, i=1,2$, and $\gamma=\gamma_{1} \cdot \gamma_{2}$, we have $N_{E / E_{3}}(\gamma)=$ $N_{E_{1} / F}\left(\gamma_{1}\right) \cdot N_{E_{2} / F}\left(\gamma_{2}\right)$. Thus we see that

$$
N_{E / E_{3}}\left(E^{\times}\right) \cap F^{\times}=N_{E_{1} / F}\left(E_{1}^{\times}\right) N_{E_{2} / F}\left(E_{2}^{\times}\right) .
$$

This equality gives us the equality of binary quadratic forms mentioned above, and the proof, which we include after the proof of Theorem 4, is a routine translation of the equality above into the language of binary quadratic forms.

Corollary. Suppose that $a, b \in F^{\times}$and $x, y \in F(\sqrt{b})$. Then $x^{2}-a y^{2} \in$ $F$ if and only if there exist $x_{i}, y_{i} \in F, i=1,2$, with

$$
x^{2}-a y^{2}=\left(x_{1}^{2}-a y_{1}^{2}\right)\left(x_{2}^{2}-a b y_{2}^{2}\right) .
$$

For a nice proof and further references for this corollary, see LeSm, Proposition 1.5] and the comments preceding it. 
Proof of Theorem 4. (2)=(3) follows from Galois theory: $\operatorname{ker}\left(1-\sigma_{i}\right)=$ $E_{i}^{\times}, i=1,2$.

$(1) \subset(4)$. Turning to multiplicative notation, we observe that

$$
\operatorname{ker}\left(1-\sigma_{1}\right)\left(1-\sigma_{2}\right)=\left\{e \in E^{\times} \mid e \cdot \sigma_{1} \sigma_{2}(e)=\sigma_{1}(e) \cdot \sigma_{2}(e)\right\} .
$$

Also $e \cdot \sigma_{1} \sigma_{2}(e)=N_{E / E_{3}}(e) \in E_{3}^{\times}$. Now assume

$$
f=e \cdot \sigma_{1} \sigma_{2}(e)=\sigma_{1}(e) \cdot \sigma_{2}(e) .
$$

Then $\sigma_{i}\left(e \cdot \sigma_{1} \sigma_{2}(e)\right)=\sigma_{1}(e) \cdot \sigma_{2}(e)=f$ for $i=1,2$. Because $\sigma_{1}, \sigma_{2}$ generate $G$, we see that $f$ is fixed by $G$. Therefore by Galois theory $f \in F^{\times}$. Hence $\operatorname{ker}\left(1-\sigma_{1}\right)\left(1-\sigma_{2}\right) \subset\left\{e \in E^{\times} \mid N_{E / E_{3}}(e) \in F^{\times}\right\}$.

$(4) \subset(3)$. Let $e \in\left\{e \in E^{\times} \mid N_{E / E_{3}}(e) \in F^{\times}\right\}$, and write $e$ as $f_{0}+$ $f_{1} \sqrt{a_{1}}+f_{2} \sqrt{a_{2}}+f_{3} \sqrt{a_{1} a_{2}}$. Then

$$
f=N_{E / E_{3}}(e)=e \cdot\left(f_{0}-f_{1} \sqrt{a_{1}}-f_{2} \sqrt{a_{2}}+f_{3} \sqrt{a_{1} a_{2}}\right) .
$$

Considering the coefficient of $\sqrt{a_{1} a_{2}}$, we obtain $f_{0} f_{3}-f_{1} f_{2}=0$.

Assume first that $f_{2} \neq 0 \neq f_{3}$. Thus $f_{0}=f_{2} t$ and $f_{1}=f_{3} t$ for some $t \in F^{\times}$. Plugging these values in our expression for $e$ gives

$$
e=\left(f_{2}+f_{3} \sqrt{a_{1}}\right) \cdot\left(t+\sqrt{a_{2}}\right) \in\left\langle E_{1}^{\times}, E_{2}^{\times}\right\rangle,
$$

where $\left\langle E_{1}^{\times}, E_{2}^{\times}\right\rangle$denotes the subgroup of $E^{\times}$generated by $E_{1}^{\times}$and $E_{2}^{\times}$. If $f_{2}=0$ then $f_{0}=0$ or $f_{3}=0$. In the first case

$$
e=\sqrt{a_{1}} \cdot\left(f_{1}+f_{3} \sqrt{a_{2}}\right) \in\left\langle E_{1}^{\times}, E_{2}^{\times}\right\rangle,
$$

and in the second case

$$
e=f_{0}+f_{1} \sqrt{a_{1}} \in E_{1}^{\times} \subset\left\langle E_{1}^{\times}, E_{2}^{\times}\right\rangle .
$$

The case $f_{3}=0$ is handled in the same way. Thus we see that

$$
\left\{e \in E^{\times} \mid N_{E / E_{3}}(e) \in F^{\times}\right\} \subset\left\langle E_{1}^{\times}, E_{2}^{\times}\right\rangle .
$$

$(3) \subset(1)$. From $E_{i}^{\times} \subset \operatorname{ker}\left(1-\sigma_{i}\right)$ we obtain $E_{i}^{\times} \subset \operatorname{ker}\left(1-\sigma_{1}\right)\left(1-\sigma_{2}\right)$. Hence $(3) \subset(1)$.

We have established $(1)=(2)=(3)=(4)$, and it remains to show that (5) is identical to the others. Observe that $(5) \subset(4)$ follows easily:

$$
\begin{aligned}
W & :=\left\{e \in E^{\times} \mid N_{E / E_{3}}(e) \in N_{E_{1} / F}\left(E_{1}^{\times}\right) N_{E_{2} / F}\left(E_{2}^{\times}\right)\right\} \\
& \subset\left\{e \in E^{\times} \mid N_{E / E_{3}}(e) \in F^{\times}\right\} .
\end{aligned}
$$


On the other hand, $(3) \subset(5)$, as follows. Let $\gamma=\gamma_{1} \cdot \gamma_{2}$ where $\gamma_{i} \in E_{i}^{\times}$, $i=1,2$. Observe that $N_{E / E_{3}} \gamma_{i}=\gamma_{i} \cdot \sigma_{1} \sigma_{2}\left(\gamma_{i}\right)=N_{E_{i} / F} \gamma_{i}$ because $\sigma_{1} \sigma_{2}$ acts on both $E_{1}$ and $E_{2}$ nontrivially. Hence we see that

$$
N_{E / F} \gamma \in N_{E_{1} / F}\left(E_{1}^{\times}\right) N_{E_{2} / F}\left(E_{2}^{\times}\right) \text {. }
$$

Therefore $\gamma \in W$. Hence all sets (1), (2), (3), (4), and (5) are identical.

Proof of the Corollary. Recall that the nonzero values of a binary quadratic form $x^{2}-d y^{2}$ for $d \in F$ form a group. Hence if $b$ is a square in $F$ our statement follows. Therefore assume that $b \in F^{\times} \backslash F^{\times 2}$.

Further observe that the set of values of quadratic forms $x^{2}-c^{2} y^{2}$, $c \neq 0$, with $x, y \in F$ is $F$ itself:

$$
f=\left(\left(\frac{f+1}{2}\right)^{2}-c^{2}\left(\frac{f-1}{2 c}\right)^{2}\right) \text { for each } f \in F
$$

Therefore if $a$ or $a b$ is a square in $F$ our statement is true as well. (Observe that if $a b$ is a square in $F$ then $a$ is a square in $F(\sqrt{b})$ and $x^{2}-a y^{2}$ represents, as values, all elements of $F(\sqrt{b})$.)

Thus assume that none of the elements $a, b, a b$ is a square in $F$. Let $a_{1}=a$ and $b=a_{2} / a_{1}$. Then since $E=E_{3}\left(\sqrt{a_{1}}\right)=E_{3}(\sqrt{a})$ we see that

$$
N_{E / E_{3}}\left(E^{\times}\right) \cap F^{\times}=\left\{x^{2}-a y^{2} \mid x, y \in E_{3}, x^{2}-a y^{2} \in F^{\times}\right\} .
$$

Also observe that $E_{3}=F\left(\sqrt{a_{1} a_{2}}\right)=F\left(a_{1} \sqrt{a_{2} / a_{1}}\right)=F(\sqrt{b})$. Finally, since $N_{E_{i} / F}\left(E_{i}^{\times}\right)=\left\{x_{i}^{2}-a_{i} y_{i}^{2} \mid x_{i}, y_{i} \in F, x_{i}^{2}-a_{i} y_{i}^{2} \neq 0\right\}$, the result follows.

\section{ACKNOWLedgements}

We thank N. D. Elkies for bringing to our attention the reference $\mathrm{O}$, which in turn had been brought to his attention by F. Lemmermeyer. Andrew Schultz would like to thank Ravi Vakil for his encouragement and direction in this and all other projects.

\section{REFERENCES}

[C] I. G. Connell. Elementary generalizations of Hilbert's Theorem 90. Canad. Math. Bull. 8 (1965), no. 6, 749-757.

[E] N. D. Elkies. Pythagorean triples and Hilbert's Theorem 90. Amer. Math. Monthly 110 (2003), no. 8, 678. 
[H] D. Hilbert. The theory of algebraic number fields. Translated from the German by Iain T. Adamson. With an introduction by Franz Lemmermeyer and Norbert Schappacher. Berlin: Springer-Verlag, 1998.

[K] E. Kummer. Über eine besondere Art aus complexen Einheiten gebildeter Ausdrücke. J. Reine Angew. Math. 50 (1855), 212-232.

[LeSc] F. Lemmermeyer and N. Schappacher. Introduction to The theory of algebraic number fields (see $\underline{\mathrm{H}}$ above).

[LeSm] D. B. Leep and T. L. Smith. Multiquadratic extensions, rigid fields and Pythagorean fields. Bull. London Math. Soc. 34 (2002), no. 2, 140-148.

[N] E. Noether. Der Hauptgeschlechtssatz für relativ-galoissche Zahlkörper. Math. Ann. 108 (1933), 411-419.

[O] T. Ono. Variations on a theme of Euler. Quadratic forms, elliptic curves, and Hopf maps. Translated and revised from the second Japanese edition by the author. Appendix 2 by M. Kida. The University Series in Mathematics. New York: Plenum Press, 1994.

[S] A. Speiser. Zahlentheoretische Sätze aus der Gruppentheorie. Math. Zeit. 5 (1919), 1-6.

[T] O. Taussky. Sums of squares. Amer. Math. Monthly 77 (1970), no. 8, 805830.

[V1] V. Voevodsky. Motivic cohomology with $\mathbb{Z} / 2$-coefficients. Publ. Inst. Hautes Études Sci., No. 98 (2003), 59-104.

[V2] V. Voevodsky. On motivic cohomology with $\mathbb{Z} / l$ coefficients. K-theory preprint archive 639. www.math.uiuc.edu/K-theory/0639/ (2003).

[We] E. Weiss. Cohomology of groups. New York: Academic Press, Inc., 1969.

[Wi] R. Wilbur. "Seed Leaves." New and collected poems. San Diego: Harcourt Brace Jovanovich, 1988, 129-130.

Department of Mathematics and Statistics, University of Missouri, Rolla, Rolla, Missouri 65409 USA

E-mail address: romand@umr.edu

Department of Mathematics, Middlesex College, University of Western Ontario, London, Ontario N6A 5B7 CANADA

E-mail address: minac@uwo.ca

Department of Mathematics, Bullding 380, Stanford University, Stanford, CALIFornia 94305-2125 USA

E-mail address: aschultz@stanford.edu

Department of Mathematics, Davidson College, Box 7046, Davidson, North CARolina 28035-7046 USA

E-mail address: joswallow@davidson.edu 